\title{
Ongoing COVID-19 Clinical Trials: A Pharmacological Perspective on Pneumonia
}

\author{
Prof. Dr. Amani E Khalifa ${ }^{1 *}$ and Asser I Ghoneim ${ }^{2}$ \\ ${ }^{1}$ Professor of Pharmacology \& Toxicology, Faculty of Pharmacy, Ain Shams University currently seconded to the Children Cancer Hospital in Egypt 57357 (CCHE \\ 57357) as the Pharmacy Consultant.
}

${ }^{2}$ Professor, Department of Pharmacology \& Toxicology, Damanhour University, Egypt

*Corresponding author: Prof. Dr. Amani E Khalifa, Professor of Pharmacology \& Toxicology, Faculty of Pharmacy, Ain Shams University currently seconded to the Children Cancer Hospital in Egypt 57357 (CCHE 57357) as the Pharmacy Consultant.

Received Date: November 26, 2020

Published Date: December 14, 2020

\begin{abstract}
COVID-19 is an ongoing global pandemic of coronavirus disease 2019 as an atypical type of viral pneumonia caused by severe acute respiratory syndrome coronavirus 2 (SARS CoV 2). Many potential pharmacological agents are currently being investigated against this disease. This article points to, and justifies, the importance of investigating the potential pharmacotherapies for COVID-19 "pneumonia", rather than the broad category of COVD-19 disease, or COVID-19 associated acute respiratory distress syndrome (ARDS).
\end{abstract}

Keywords: COVID-19; Trials, Pharmacotherapy; Perspective; Pneumonia

\section{Introduction}

Coronavirus disease 2019; the current pandemic, is a newly emerged viral pneumonia that was named COVID-19 by the WHO on February 11th, 2020 [1]. The causative agent of this viral pneumonia is the SARS-CoV-2 which was found to be closely similar to SARS-CoV with about $80 \%$ sequence identity [2]. Although the pathogenesis of COVID-19 disease including the mechanisms of antigen presentation is poorly understood, the high similarity between SARS-CoV-2 and SARS-CoV was reflected in the clinical course of the disease where the virus typically attacks the lungs in certain vulnerable populations in three phases; viral replication, immune hyper-reactivity, and pulmonary destruction [3].

\section{Clinical studies of COVID-19 disease}

The database registry of "ClinicalTrials.gov" run by the United States National Library of Medicine at the National Institutes of Health includes all privately and publically funded clinical studies conducted in 211 countries around the world. Observational and interventional studies from Egypt (148 studies) and the rest of the world (4019 studies) are taking place [4]. Among such running clinical trials is our team's principal investigation of the roles of Egyptian pharmacists in combating this disease that is available at: https://clinicaltrials.gov/ct2/show/NCT04374513

Specifically, "Clinicaltrials.gov" database registry classified the overall listed clinical studies related to COVID-19 into many categories according to the targeted condition. Three categories are most prominent; one major category targeting the COVID-19 disease condition in general including mild, moderate and/or severe disease with or without evidence of pneumonia, another category of clinical studies targeting confirmed COVID-19 pneumonia, and a third category targeting COVID-19 associated ARDS, with some overlap that might exist among these categories. Among the updated total of 4019 studies on COVID-19 generally, the refined search strategy yielded only 747 on pneumonia in COVID-19 [5].

From a pharmacological point of view, it is important to focus on the second category of clinical studies targeting COVID-19 pneumonia rather than COVID-19 associated ARDS or the broad COVID-19 disease categories for many reasons. First, the majority 
of COVID-19 patients exhibit mild to moderate symptoms, and only $15 \%$ of patients progress to severe pneumonia cases within which about 5\% eventually develop ARDS, septic shock, and/or multiple organ failure $[3,6]$. So, the disease can be tolerated by most patients with a positive outcome of viral load reduction/ clearance, followed by receding of inflammation and in this population, the benefit/risk ratio of any pharmacological intervention may not be ethically justified and may also negatively affect their otherwise capable immune system function. The category of clinical trials targeting the COVID-19 disease condition in general including mild, moderate, and/or severe disease, therefore may expose many cases to unneeded and unknown risk due to the medications' side effects/toxicities in this novel disease. Also, the same pharmacological interventions may not be appropriate for all levels of disease severity given the reported multistage pathophysiology of CoVs discussed earlier. On the other hand, patients experiencing ARDS develop overwhelming lung hyper-inflammatory cytokine storm and acute respiratory failure; a critical and late condition in the course of COVID-19 disease that might not allow for a completely successful intervention. Therefore, in clinical trials targeting COVID-19 associated ARDS, reaching a safe and effective pharmacotherapy would be utterly difficult. Besides, treatment before the onset of ARDS could be a wise decision since the overall prognosis of ARDS is poor, with mortality rates of approximately $40 \%$ [7], and even if patients survive, they might face other life-long physical and psychological consequences after exhausting their economic and national healthcare systems.

It is also important to focus on the second category of clinical studies targeting COVID-19 pneumonia rather than COVID-19 associated ARDS or the broad COVID-19 disease category since the available clinical data indicates that the majority of COVID-19 patients had pneumonia while only small percentage had ARDS. In a Chinese cohort of 1,099 patients with COVID-19, 93.6\% were hospitalized, $91.1 \%$ had pneumonia, 5.3\% were admitted to the ICU, $3.4 \%$ had ARDS and only $0.5 \%$ had acute kidney injury [8]. In addition, a retrospective study that included 357 patients with ARDS who did not have chronic kidney disease or acute kidney injury before ARDS presentation reported that pneumonia was the cause of ARDS in $83 \%$ of patients [9]. Therefore, safe and effective treatment for COVID-19 pneumonia is necessary, particularly if targeted to the cases with potential bad prognosis whose immune system could not otherwise overcome such illness without intervention (Figure 1).

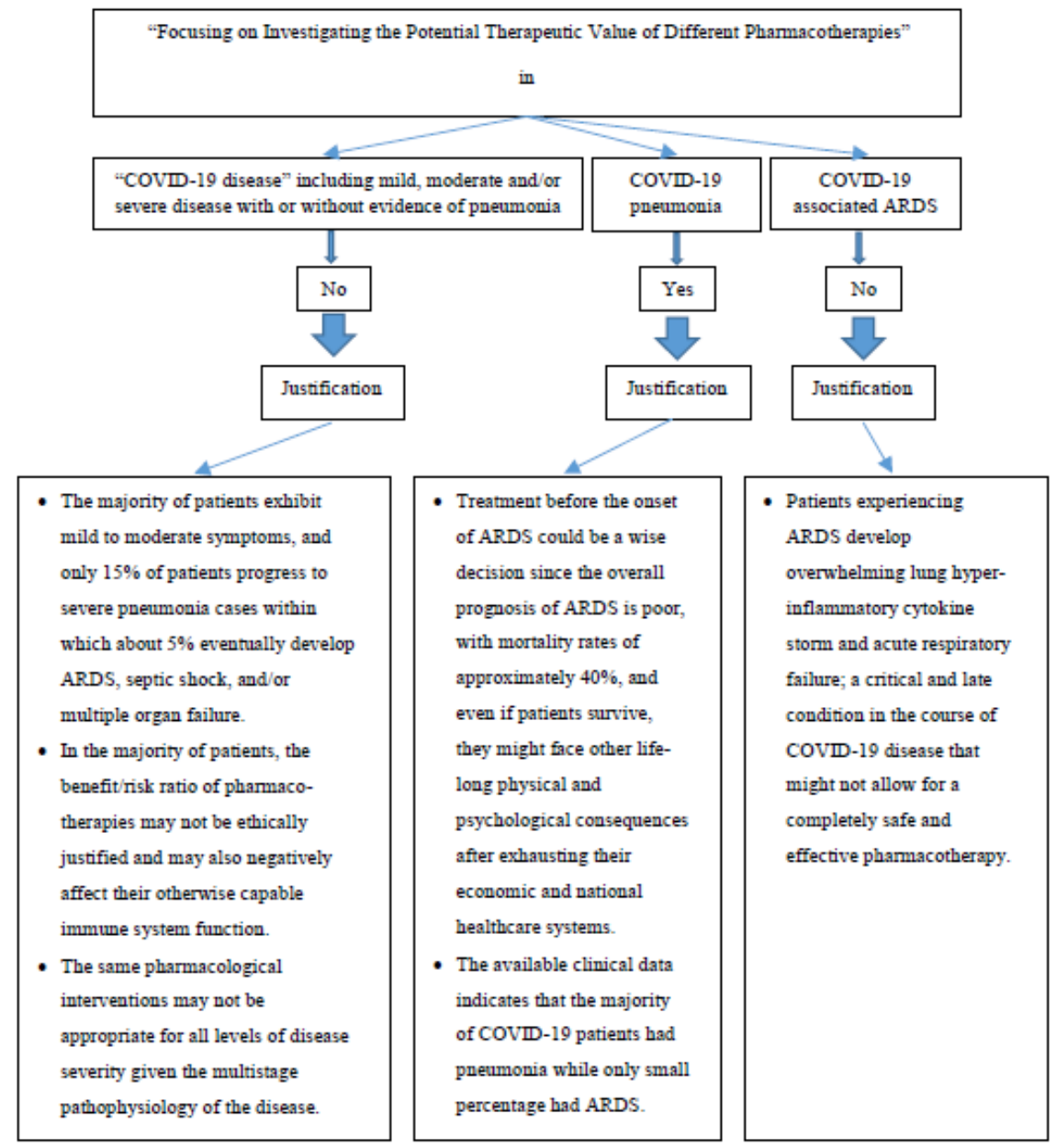

Figure 1: Ongoing COVID-19 trials: A pharmacological perspective on pneumonia 


\section{Conclusion}

Finally, it is important to focus on trials investigating potential pharmacotherapies against COVID-19 pneumonia in particular rather than the broad category of COVID-19 disease (including mild, moderate, and/or severe disease with or without evidence of pneumonia), or those of COVID-19 associated ARDS. In all cases, it is suggested that interventions in COVID-19 pneumonia patients be limited to those with potential poor prognosis and hence it is important to devote more attention to developing and advancing clinical prediction rules to more objectively predict outcomes of COVID-19 pneumonia.

\section{Declarations}

\section{Ethical Approval}

Ethical approval status of this opinion article was exempt from review by the Research Ethics Committee of the Faculty of Pharmacy at Damanhour University.

\section{Consent to participate and to Publish}

The two contributing authors gave their consent to participate in and to publish this manuscript.

\section{Authors contributions}

AK and AG conceived, designed and performed research. AK and $A G$ wrote and approved the manuscript, and all data were generated in-house and no paper mill was used.

\section{Competing interests}

The two authors declared no potential conflicts of interest with respect to the research, authorship, and/or publication of this opinion article.

\section{Availability of data and materials}

Transparent data supporting those reported in the manuscript are included such as hyperlinks to publicly archived datasets as clinicaltrials.gov generated during the study.

\section{Acknowledgment}

The current research did not receive any grants from funding agencies in the public or commercial sectors.

\section{Conflict of Interest}

No conflict of interest.

\section{References}

1. Zhou P, Yang XL, Wang XG (2020) A pneumonia outbreak associated with a new coronavirus of probable bat origin. Nature 579: 270-273.

2. Hirano T, Murakami M (2020) COVID-19: A new virus, but a familiar receptor and cytokine release syndrome. Immunity 52(5): 731-733.

3. Huang C, Wang Y, Li X (2020) Clinical features of patients infected with 2019 novel coronavirus in Wuhan, China. Lancet 395: 497-506.

4. US National Library of Medicine (2020a) Clinicaltrials.gov.

5. US National Library of Medicine (2020b) Clinicaltrials.gov.

6. Xu Z, Shi L, Wang Y (2020) Pathological findings of COVID-19 associated with acute respiratory distress syndrome. Lancet Respir Med 8(4): 420422.

7. Lewis SR, Pritchard MW, Thomas CM, Smith AF (2019) Pharmacological agents for adults with acute respiratory distress syndrome. Cochrane Database Syst Rev 7(7): CD004477.

8. Guan WJ, Ni ZY, Hu Y (2020) Clinical characteristics of coronavirus disease 2019 in China. N Engl J Med 382: 1708-1720

9. Panitchote A, Mehkri O, Hasting A (2019) Factors associated with acute kidney injury in acute respiratory distress syndrome. Ann Intensive Care 9(1): 74 . 